\title{
Anti-slip control research for the electric vehicle of in-wheel motor drive
}

\author{
Jian-qiang $\mathrm{SU}^{*}$ \\ Department of Automation, Institute of electric power, Inner Mongolia University of Technology, 010080 Hohhot, CHINA
}

\begin{abstract}
In-wheel motor drives electric vehicles are becoming more and more widely used due to their unique advantages. This paper addresses the problem of in-wheel motor drive electric vehicle wheels slipping on low-attached roads. An active disturbance rejection controller is designed to control the inwheel motor torque and prevent the wheel slipping. The co-simulation is carried out between the adams and Matlab, and the results of simulation demonstrated that the controller which can prevent the wheel slipping effectively was perfect. The most important is that the controller can be implemented easily.
\end{abstract}

\section{Introduction}

In-wheel motor drive electric vehicle has superiorities of flexible system collocation and fleetly driving speciality, which is an important develop direction of electric vehicle. The mechanical connection between the wheels is abolished, and the wheel is a self-governed system in the vehicle. If the motor torque is not limited when the vehicle is on the low-adhesion surface or the wheel is suspended, the wheel may be slipped which affects the traffic safety. The anti-skid control is adopted to control the wheel speed in a certain range by adjusting the inwheel motor torque in order to ensure the safety of the vehicle, which also can advance the longitudinal and lateral characteristics of the tire.

In this paper, the active disturbance rejection control is applied to the anti-slip control of the wheel. For one thing, the vehicle dynamics system is considered to be a large disturbance and uncertain system which need a nonlinear controller, and for another thing, the achievability of the control algorithm in real vehicle application is guaranteed.

\section{The configuration of the Vehicle}

In this paper, the in-wheel motor drive electric vehicle has four motors which have independent controller, and the vehicle controller, batteries management system and the motor controllers are communicated by CAN bus. The vehicle electric brake can be brought about and the resistance can emit energy when DC bus voltage is beyond the warning value. The configuration is in the Fig.1.

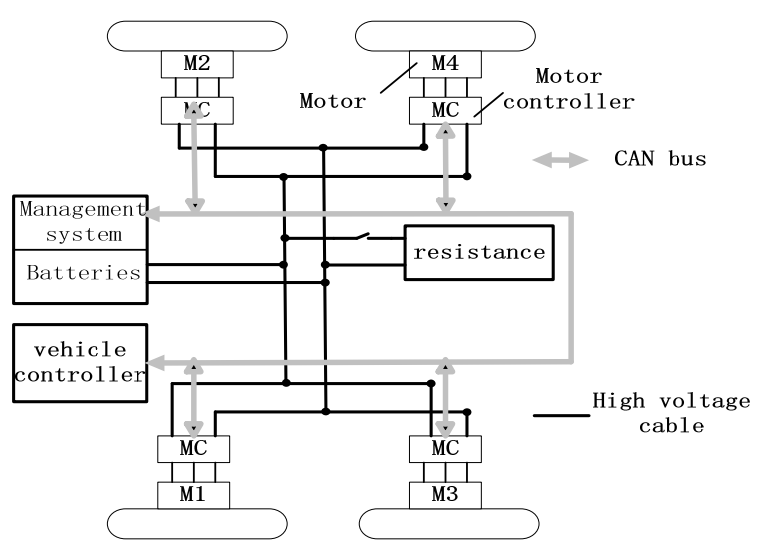

Fig. 1. The configuration of the vehicle

\section{The Wheel dynamics model}

The wheel system can be used as an independent system in the anti-slip control. The dynamics and tire model of the vehicle wheels are analyzed below.

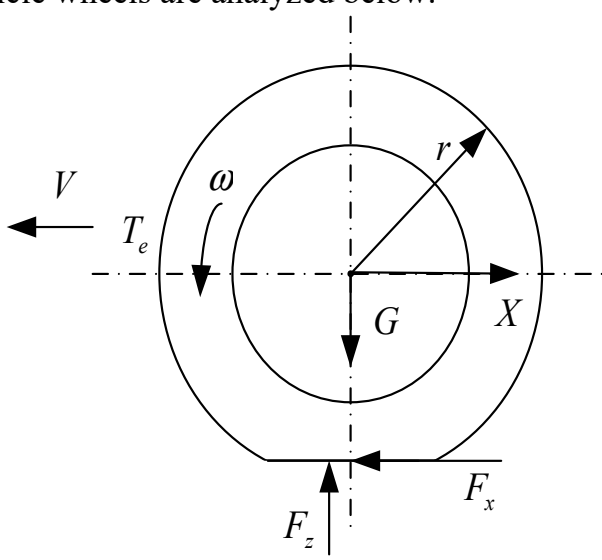

Fig. 2. The wheel dynamics

\footnotetext{
* Corresponding author: sujianqiang1983@163.com
} 
The wheel model of the vehicle on the horizontal road is:

$$
\left\{\begin{array}{c}
J \dot{\omega}=i T_{e}-\mu(\lambda) F_{z} r-f F_{z} r \\
F_{z}=\frac{1}{4} m g+\Delta F_{z} \\
\lambda=\frac{(\omega r-V)}{\omega r}
\end{array}\right.
$$

Where: $m$ is the total mass of the vehicle; $i$ is the ratio between the motor and the wheel; $J$ is the moment of inertia of the wheel; $V$ is the vehicle speed; $\lambda$ is the wheel slip rate; $r$ is the wheel radius; $\omega$ is the wheel angular velocity; $\mu(\lambda)$ is the adhesion coefficient of the wheel; $f$ is the rolling resistance coefficient of the wheel; $T_{e}$ is the electromagnetic torque of the motor; $F_{z}$ is the load of the wheel; $\Delta F_{z}$ is the dynamic load of the wheel.

The torque transient response performance of the inwheel motor is several times faster than the transient response of the wheel, and the torque model of the motor can be simplified as a first-order dynamic system model: $\dot{T}_{e}=\frac{1}{\tau}\left(T_{e_{-r e f}}-T_{e}\right)$

Where: $T_{e_{-} r e f}$ is the motor reference torque, $\tau$ is the motor torque response time constant.

If $x_{1}=\omega, x_{2}=\dot{\omega}, \mathrm{u}=T_{e_{-r e f}}$, the system's state equation is:

$$
\left\{\begin{array}{c}
\dot{x_{1}}=x_{2} \\
\dot{x_{2}}=\frac{i}{J \tau} e^{-\frac{t}{\tau}} u-\frac{\mu(\lambda) F_{z} r+f F_{z} r}{J}
\end{array}\right.
$$

The wheel load includes static load and dynamic load, and the dynamic load is caused by the acceleration or deceleration of the vehicle and the uneven road surface, and the inertia of the wheel is caused to change during running because of the resistance viriety. The system is an non-linear time-varying system obviously.

The tire transmits power through the friction force of the ground. When the tire is attached to the ground, the maximum value of the longitudinal force is limited by the frictional relation between the tire and the road surface. If the wheel driving force is greater than the maximum longitudinal force on the ground, the tire is no longer Attached to the ground, the wheels are will be sliping and in an unstable state. Anti-slip control keeps the wheel as close to the optimal slip rate $\lambda_{d}$ as possible, allowing the ground to provide maximum driving force. The curve between slip ratio and ground adhesion coefficient is in the Fig.3.

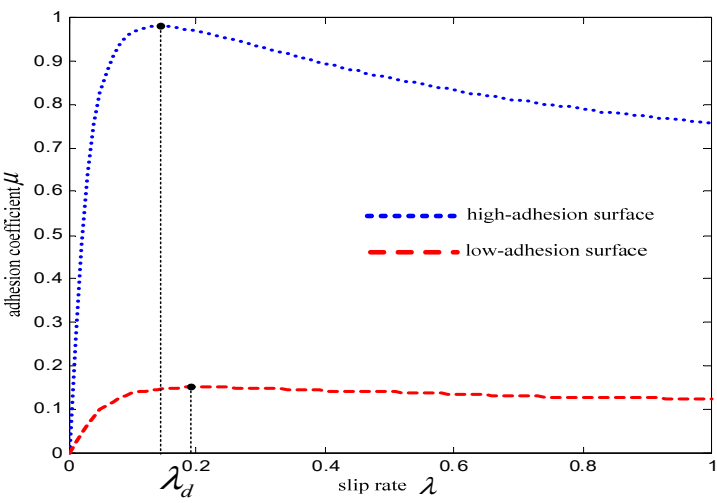

Fig. 3. The curve between slip ratio and ground adhesion coefficient

In this paper, the target slip rate is taken as the settled slip rate in the slip control process.

\section{4 anti-slip control}

The active disturbance rejection control technology considers the unknown part and unknown disturbance in the controlled model as the total disturbance of the system. The extended state observer (ESO) is used to estimate and compensate the total disturbance of the system in real time. The differentials state of the system is trace controlled by tracking differentiator (TD). Nonlinear control of "small error large gain, large error small gain" is achieved by the action of nonlinear state error feedback (SEF) control rate.

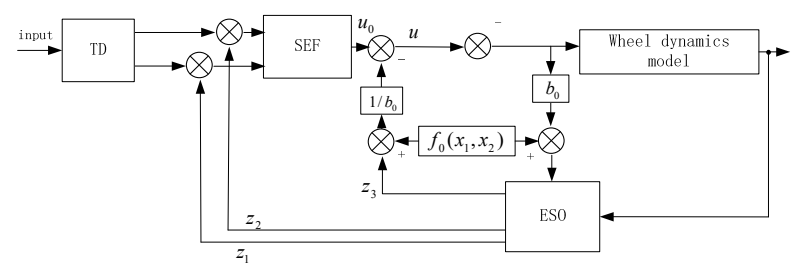

Fig. 4.The active disturbance rejection control system

The system can be converted to the state equation :

$$
\left\{\begin{array}{c}
\dot{x_{1}}=x_{2} \\
\dot{x_{2}}=b u+F(t)
\end{array}\right.
$$

Where: $b_{0}=\frac{i}{J \tau}, F(t)$ is the total disturbance of system.

The vehicle is interfered by diversified uncertain factor, which can be observed by ESO.

$$
\left\{\begin{array}{c}
e(t)=z_{1}(t)-x_{1}(t) \\
\dot{z}_{1}(t)=z_{2}(t)-\beta_{1}(e(t)) \\
\dot{z}_{2}(t)=z_{3}(t)-\beta_{2} f a l\left(e(t), a_{2}, \delta\right)+b u \\
\dot{z}_{3}(t)=-\beta_{3} \operatorname{fal}\left(e(t), a_{3}, \delta\right)
\end{array}\right.
$$

Where : $z_{1}(t)$ is the observe value of wheel angular velocity $x_{1}(t) ; z_{2}(t)$ is the observe value of wheel angular acceleration $x_{2}(t) ; z_{3}(t)$ is the observe value of disturbance $F(t)$.

$f a l\left(e, a_{2}, \delta\right)=\left\{\begin{array}{cc}|e|^{a} \operatorname{sign}(e) & |e|>\delta \\ \frac{e}{\delta^{1-a}} & |e| \leq \delta\end{array}\right.$ 
From $\omega=\frac{V}{r\left(1-\lambda_{d}\right)}$, the given angular velocity $\omega_{\text {ref }}$ can be calculated which is the input signal, and the input signal transition process is designed by TD. The output signals are $\omega_{\text {ref } 1}$ and $\omega_{\text {ref } 2}$.

$$
\left\{\begin{array}{c}
\omega_{r e f 1}(k+1)=\omega_{r e f 1}(k)+h \omega_{r e f 2}(k) \\
\omega_{r e f 2}(k+1)=\omega_{r e f 2}(k)+h f s t(\cdot)
\end{array}\right.
$$

Where :The most rate function is

$$
f \operatorname{st}(\cdot)=-r * \operatorname{sat}(g(k), d), d=h r, d_{0}=d h \text {. }
$$

Where :

$$
\operatorname{sat}(g(k), d)=\left\{\begin{array}{cc}
\operatorname{sign}(g(k)) & |g(k)|>d \\
\frac{g(k)}{d} & |g(k)| \leq d
\end{array}\right.
$$

Where $: g(k)=$

$$
\left\{\begin{array}{c}
\omega_{r e f 2}(k)-\operatorname{sign}(y(k)) * \frac{d-\sqrt{8 r|y(k)|+d^{2}}}{2} \\
\omega_{r e f 2}(k)+y(k) / h
\end{array}\right.
$$

Where :

$$
y(k)=\omega_{\text {ref } 1}(k)-\omega_{\text {ref }}(k)+h \omega_{\text {ref } 2}(k)
$$

Where $: h$ is the sampling period; $r$ is the parameter of tracking speed.

In order to simplify the operation, a second order system is used for the SEF.

$$
\left\{\begin{array}{c}
e_{1}=\omega_{\text {ref } 1}-z_{1}(t) \\
e_{2}=\omega_{\text {ref } 2}-z_{2}(t) \\
u_{0}=\beta_{01} f a l\left(e_{1}, a_{1}, \delta_{1}\right)+\beta_{02} f a l\left(e_{2}, a_{2}, \delta_{2}\right)
\end{array}\right.
$$

The system is changed to a type of integralors tandem, and control amount is :

$$
u_{d}=u_{0}-\frac{1}{b_{0}} z_{3}(t)
$$

After the anti-slip control, the reference torque of the in-wheel motor is $T_{e_{-} r e f}=\min \left(T_{r e f}, u_{d}\right)$, where $T_{\text {ref }}$ is the driver throttle the corresponding motor torque. When the vehicle is running on a high-adherent road, the actual slip ratio of the wheel is very small, and the antiskid control calculation must be greater than the throttle given torque, and the motor reference torque $T_{e_{-} r e f}=T_{r e f}$. At this time, the reference torque of the in wheel motor is given by the accelerator pedal, and the anti-slip function does not work. When driving on the low adhesion surface, the ground cannot pass the power given by the driver. The anti-slip control controls the wheel slip rate in the optimal range to ensure that the vehicle accelerates with maximum power performance.

\section{Simulation}

In order to verify the effectiveness of the control algorithm, a co-simulation test was performed on a lowattachment road surface. The vehicle model is found in the adams, in the same time the motor model and controller are found in the Matlab/Simulink. The cosimulation is carried out between the adams and Matlab.

The figure shows a straight line driving acceleration simulation that the vehicle was tested on the F-class road surface $u_{\max }=0.3$. Without the control, the driving torque is directly added to the wheels. When the wheel speed is low, the motor's driving torque is large. As shown in Fig. 5(b), the slip ratio of the wheels reaches 0.6 at the maximum, and the wheels are in an unstable state. As the speed increases, the motor is in constant power control. Although the torque drops as shown in Fig. 5(c), the slip rate decreases, and the track of the vehicle is shown in Fig. 5(d), which seriously deviates from driving purpose.

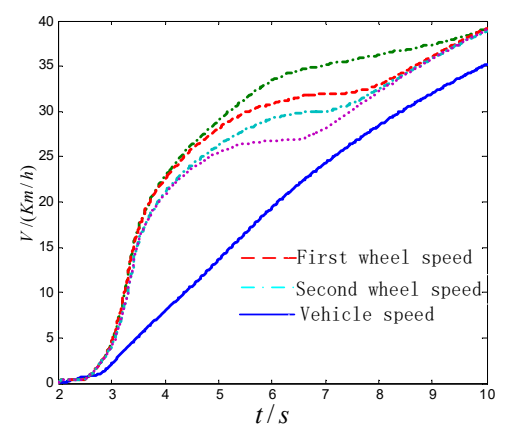

Fig. 5. (a)Vehicle speed and wheels speed

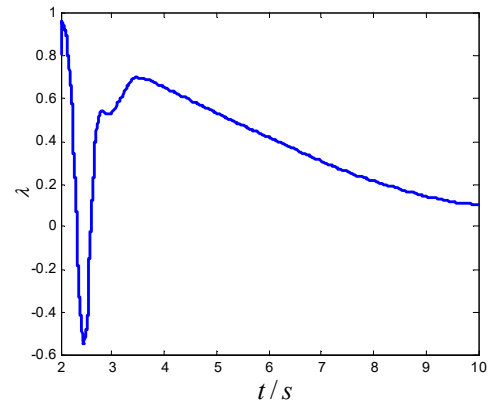

Fig. 5. (b) The slip rate of first wheel

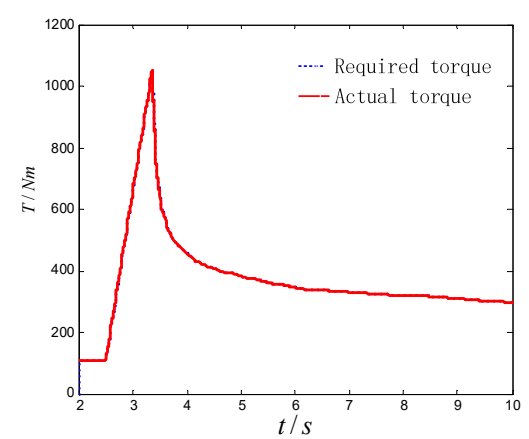

Fig. 5. (c) The torque of the motor 


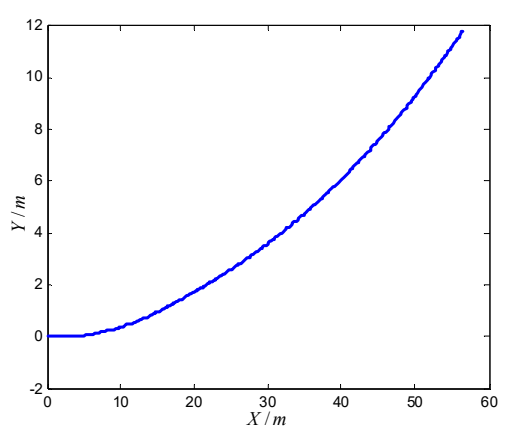

Fig. 5. (d) The track of vehicle running on the low-adhedion surface without control

After the anti-slip control is adopted, the actual slip ratio of the wheel can be quickly controlled at 0.2 in Fig. 6(b) when the reference slip ratio is 0.2. As shown in Fig. 6(a), the vehicle has better performance than when there is no control. The vehicle maintains the stability of straight running, as shown in Fig. 6(d). The torque is relatively stable. At that time $t=7.6 \mathrm{~s}$, the anti-skid control is exited and the motor torque is given by the drive in Fig. 6(c).

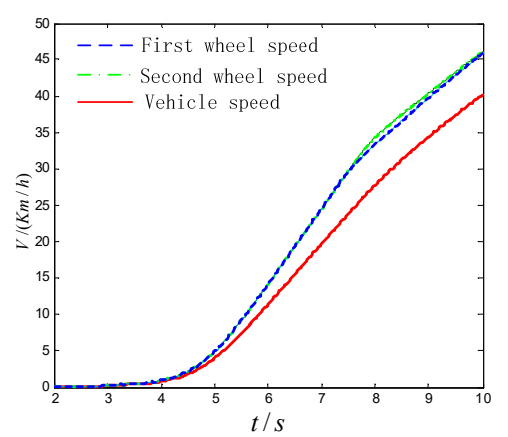

Fig. 6. (a)Vehicle speed and wheels speed

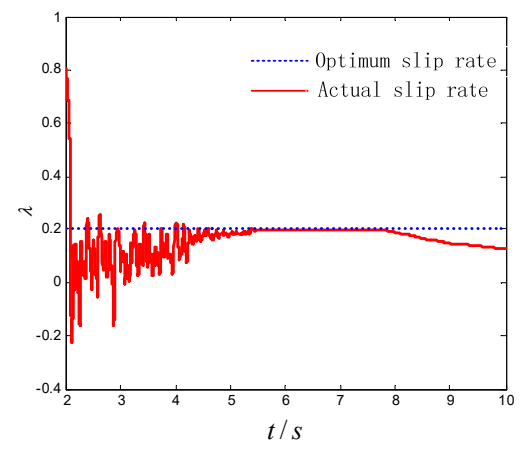

Fig. 6. (b) The slip rate of first wheel

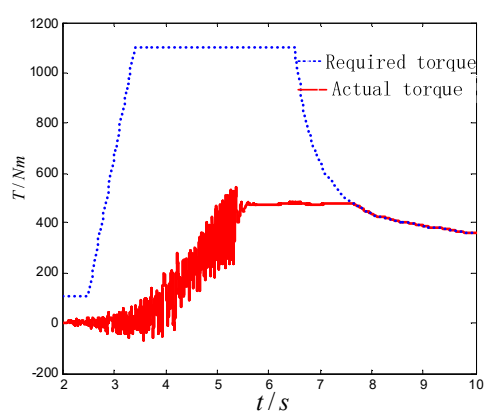

Fig. 6. (c) The torque of the motor

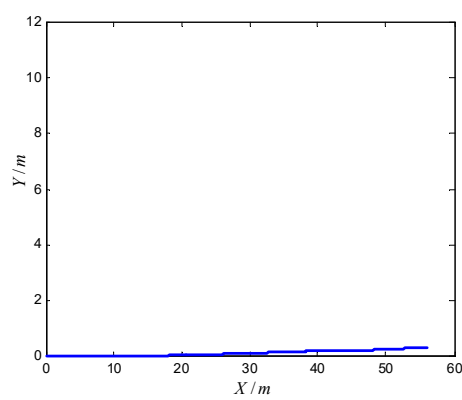

Fig. 6. (d) The track of vehicle running on the low-adhedion surface with control

\section{Conclusion}

The joint simulation test shows that the active disturbance rejection controller is designed to prevent the wheel slipping effectively. The controller is simple in design and easy to implement.

\section{References}

1. HE Ren, ZHAN GRui-jun. Research and Development of in-Wheel Motor Drive Technology, Journal of Chongqing University of Technology, 29:10-18 (2015)

2. W.-S. Lin, L.-H. Chang and P.-C. Yang. Adaptive critic anti-slip control of wheeled autonomous robot, IET Control Theory Appl, 1:51-57 (2007)

3. Kyoungseok Han, Mooryong Choi, Byunghwan Lee, Seibum B. Choi. Development of a Traction Control System Using aSpecial Type of Sliding Mode Controller for Hybrid 4WD Vehicles, IEEE Tran. Vehicular technology, 67:264-274, (2018)

4. Zhiwu Huang, Zheng Xu. Sliding Mode Control for Urban Railway Anti-Slip System Based on Optimal Slip Ratio Estimation with Forgetting Factor Recursive LeastSquares, Proceedings of the 36th Chinese Control Conference Dalian:9502-9507, (2017)

5. Lin W S, Chang L H, Yang P C. Adaptive Critic Anti-slip Control of Wheeled Autonomous Robot, IET Control Theory, 1:51-57(2007)

6. Xibo Yuan, Jiabin Wang. Torque Distribution Strategy for a Front- andRear-Wheel-Driven Electric Vehicle, IEEE Trans on vehicular technology, 61: 3365-3374(2012)

7. GAO Zhi-qiang. On the foundation of active disturbance rejection control, Control Theory \& Applications, 30:1498-1510(2013)

8. Han Jingqing. Active Disturbance Rejection Control Technology, Beijing: National Defense Industry Press (2008) 